\title{
Congestion Control to Achieve Optimal Broadcast Efficiency in VANETs
}

\author{
Fei Ye \\ Student Member, IEEE \\ University of Washington \\ Seattle, WA 98195 \\ fye@u.washington.edu
}

\author{
Raymond Yim \\ Member, IEEE \\ Mitsubishi Elect. Research Labs \\ Cambridge, MA 02139 \\ yim@merl.com
}

\author{
Jinyun Zhang \\ Fellow, IEEE \\ Mitsubishi Elect. Research Labs \\ Cambridge, MA 02139 \\ jzhang@merl.com
}

\author{
Sumit Roy \\ Fellow, IEEE \\ University of Washington \\ Seattle, WA 98195 \\ sroy@u.washington.edu
}

\begin{abstract}
In a vehicular network, every vehicle broadcasts update messages that contain location and speed information periodically to its one hop neighbors. The broadcast efficiency measures the average rate at which a vehicle receives these packets from any of its neighbors. As the node density increases, heightened interference lowers broadcast efficiency if congestion control mechanism is not used. In this paper, we analyze the broadcast efficiency under Rayleigh fading channel, and provide congestion control and power control strategies that maximize the efficiency. A worst-case guaranteed strategy achieving at least $95 \%$ of the optimal is also provided for cases when the network nodes have high mobility. Ns-2 simulations show that our analytical results accurately predict the system dynamic.

Index Terms-Congestion Control, Broadcast Efficiency,
\end{abstract} Rayleigh Fading, Capture Effect, Power Control, Node Density

\section{INTRODUCTION}

Vehicular ad hoc network (VANET) is a promising form of mobile ad hoc network, in which vehicles are equipped with radios for inter-vehicle communication [1]-[6]. As an important part of intelligent transportation system, VANET enables run-time vehicle-to-vehicle $(\mathrm{V} 2 \mathrm{~V})$ and vehicle-toinfrastructure (V2I) message exchange, for better safety, fuel efficiency, comfort and entertainment. At its basics, road safety can be greatly improved if the onboard communication unit could provide drivers with a greater awareness about their surrounding environments. In fact, the Society for Automotive Engineers (SAE) [7] and the IEEE WAVE standard [8] have defined a special type of "heart beat" message that should be periodically broadcast by vehicles to inform one-hop neighbors of their locations, velocities and directions of travel, etc.

When every node in the network has messages to send, a good measure of broadcast performance is the average rate at which any particular node receives packets successfully from any source ${ }^{1}$. We call this the broadcast efficiency. Many simulation-based works have been conducted to investigate similar performance metric. For example, one hop broadcast reception probability is studied in [2], [9]. Through these simulations, one observes the relationship between node density and traffic load for maximum broadcast performance. Also, simulations in [5] demonstrate that VANETs having the same

\footnotetext{
${ }^{1}$ This is equivalent to the average number of nodes to which a node successfully delivers its transmission in a unit time.
}

communication density might have similar reception probability versus distance for a specific broadcast packet. [6] derives a protocol that varies transmission power so that the beaconing load perceived by each vehicle in carrier sensing does not violate a maximum beaconing load regardless of node density. [10] studies the unsaturated performance of a channel with two categories of services using distance based reception model without capture capability. Finally, mathematical expressions for broadcast performance were obtained empirically through ample simulations and least-square curve fitting in [11].

Network transmission efficiency has also been studied extensively in wireless local area networks [12]-[15]. While the performance metric is not the same as broadcast efficiency since these networks have only a single dedicated received node per transmission, these studies shine light on wireless network performance. For example, [13], [14] show that optimal contention window size should scale with the total number of nodes in a WLAN. In [12], the capture effect based on receive signal to interference plus noise ratio (SINR) is shown to be able to greatly increase reception probability.

In this paper, we introduce and study broadcast efficiency under fading and interference. To the best of our knowledge, this is the first mathematical characterization on broadcast performance of VANETs with fading channel and capture effect. This mathematical analysis is of significant theoretical importance, since it provides guidance to VANET system designs without the need of extensive simulations. Through ns-2 simulations over highway scenarios, we validate our theoretical analysis, and show that our analysis closely resembles the results from network simulations. The theoretical analysis reveals the following key insights to broadcast efficiency:

- Let $\alpha$ be the path loss exponent and $p_{0}$ be the transmission power, then, with all other system parameters fixed, the same broadcast efficiency can be achieved by scaling $p_{0}^{1 / \alpha}$ inversely proportionally to node density. This has direct implication to optimal power control in VANETs, and it has never been observed previously.

- Contrary to common assumptions [5], [13], [14], the same broadcast efficiency cannot be achieved by simply scaling the transmission probability inversely proportional to the node density. The complete optimization equation is 
provided in this paper.

If node density is known, this paper provides congestion control mechanism to achieve the optimal broadcast efficiency. However, in VANET, the exact node density is difficult to estimate in runtime due to the dynamic nature. In this case, we show a significant result that, even with loose lower and upper bounds on the density that may be an order of magnitude apart, it is possible to derive a contention control algorithm that guarantees a worst case performance that is no less than $95 \%$ of the optimal broadcast efficiency as if the exact node density is known.

\section{SySTEM MODEL}

We consider a vehicular network consisting of a number of packet generating nodes (vehicles) that broadcast messages to their neighbors. We assume that the nodes always have packets waiting in their outgoing queues, and all packets have the same size of $L$ bits, and a fixed modulation and coding is used to transmit the packets at $R$ bits/sec. We perceive that the nodes are present in stretch of straight highway, and we omit lane information for simplicity. Hence, nodes are aligned in a onedimensional linear space. The inter-node spacing is assumed to be exponentially distributed with mean distance $1 / \lambda$.

The system has a single broadcast channel that is shared amongst all nodes. Before sending a packet, the node first senses whether the channel is free. If it is so, the node broadcasts a packet with probability $c$ regardless of the actions of the other nodes in the system. When a node transmits a packet, it transmits the packet without interruption for $T_{\mathrm{tx}}$ seconds. If the node chooses to not transmit when a channel is free, it waits for $T_{\text {idle }}$ seconds before it senses the channel again. Finally, if the channel is not free, the node attempts to decode the packet, and senses the channel again afterwards. This procedure is very similar to $p$-persistent Carrier-Sense Multiple Access (CSMA) [16].

The node broadcasts packets with transmission power $p_{0}$. We assume that the wireless channels between any two nodes exhibit Rayleigh fading characteristics, and the path loss exponent is $\alpha$. Let $d$ be the distance between a source and a receiver, and $S$ be the receive power of the transmission from a single source, then $S$ follows an exponential random variable with mean $p_{0} d^{-\alpha}$, which has the following probability distribution function (pdf):

$$
g(s)=\frac{1}{p_{0} d^{-\alpha}} \exp \left(-\frac{s}{p_{0} d^{-\alpha}}\right), \quad \forall s \geq 0 .
$$

The receiver has only single packet reception capability, and can decode the packet successfully if and only if its receive SINR exceeds a threshold:

$$
\frac{s}{\sum_{i=1}^{\infty} I_{i}+n_{0}} \geq z
$$

where $n_{0}$ is the noise power, $\sum_{i=1}^{\infty} I_{i}$ is the total interference power from transmission of neighboring nodes, and $z \geq 1$ is a threshold that depends on the modulation and coding used for the packet transmission.

\section{THEORETICAL ANALYSIS}

\section{A. Single Transmission with Background Interference}

In this subsection, we first analyze the receive power of a single transmission over a fading channel when the distance between the source and destination is a random variable. We then study the probability that a node receives a specific packet successfully when other nodes transmit interfering packets simultaneously with probability $c$. Finally, we show, when a node transmits a packet, the expected number of nodes that receive and decode the specific packet successfully.

Let the receiving node be located at the origin of a onedimensional axis, and we fold the space so that the transmitting nodes are located only in the positive axis. Hence, effectively, the inter-node spacing is exponentially distributed with mean distance $1 / 2 \lambda$. Furthermore, since each node independently broadcasts a packet with probability $c$, the distribution of the transmitting nodes is a Poisson point process with mean $2 \lambda c$. To facilitate the analysis, we first consider that transmitting nodes are located within $d_{m}$ meters from the receiving node. The value of $d_{m}$ is chosen to be sufficiently large so that a packet sent from any node further than $d_{m}$ away is received with negligible power (i.e., $p_{0} d_{m}^{-\alpha}<<z n_{0}$ ), and we will eventually set $d_{m} \rightarrow \infty$ for our final result.

The following Lemma gives a straightforward result that shows the distribution of receive power due to a single transmitter. Proofs are omitted or only key steps are shown in this paper due to space constraint.

Lemma 1: The pdf $f_{S}(s)$ and cumulative distribution function (cdf) $F_{S}(s)$ of the reception power of a single transmitter that is uniformly distributed within $\left[0, d_{m}\right]$ are given by

$$
\begin{aligned}
& f_{S}(s)=\frac{1}{d_{m} p_{0}} \int_{0}^{d_{m}} x^{\alpha} \exp \left(-p_{0}^{-1} x^{\alpha} s\right) \mathrm{d} x, \\
& F_{S}(s)=1-\frac{1}{d_{m}} \int_{0}^{d_{m}} \exp \left(-p_{0}^{-1} x^{\alpha} s\right) \mathrm{d} x .
\end{aligned}
$$

To compute the probability of successfully receiving a specific packet, we need to characterize the distribution of the total interference. From (3) and (4), it can be shown that the distribution of the receive power is heavy tail. Hence, for analytical tractability, we will approximate the total interference by the strongest interfering component.

The following Lemma shows the probability that a node receives a specific packet successfully when the source of the packet is uniformly distributed in $\left[0, d_{m}\right]$, while other nodes transmit interfering packets with probability $c$.

Lemma 2: Consider a network with background interference coming from nodes that are spatially Poisson distributed along a single dimension in $\left[0, d_{m}\right]$ with mean $2 \lambda$. Each node independently transmits an interfering packet with probability $c$. Then, the probability that a node receives and decodes a packet successfully from a specific source node that is uniformly distributed in $\left[0, d_{m}\right]$ is

$$
P_{\text {s-spec }}=\int_{z n_{0}}^{\infty} P(\operatorname{succ} l s) f_{S}(s) \mathrm{d} s,
$$


where $f_{S}(s)$ is the pdf of the receive power of a single transmission given by (3), and $P(\operatorname{succ} \mid s)$ is the probability of successfully decoding a packet with receive power $s$, which is given by

$$
P(\operatorname{succ} \mid s)=\exp \left(-2 \lambda c d_{m}\left(1-F_{S}\left(z^{-1} s-n_{0}\right)\right)\right), \forall s \geq z n_{0} .
$$

Proof: Compute the probability that receive SINR is higher than $z$ and use order statistics to obtain interference.

The following key result shows, when a node broadcasts a packet, the expected number of nodes that receive and decode the specific packet successfully. Note that the result applies to an infinitely large one-dimensional network, and it no longer depends on $d_{m}$.

Theorem 1: Consider a network having Poisson distributed nodes with mean $\lambda$, and each node independently transmits a packet with probability $c$. Let $N$ be the number of nodes that decode a packet originated from the same transmission successfully, then

$E[N]=\frac{(1-c)}{c z^{1 / \alpha}}\left(1-\exp \left(-2 c \lambda\left(p_{0} / n_{0}\right)^{1 / \alpha} \Gamma\left(1+\frac{1}{\alpha}\right)\right)\right)$,

where $\Gamma(x)=\int_{0}^{\infty} t^{x-1} e^{-t} \mathrm{~d} t$ is the Gamma function.

Proof: It is easy to see that $E[N]=\lim _{d_{m} \rightarrow \infty} 2 \lambda(1-$ c) $d_{m} P_{\mathrm{s}-\mathrm{spec}}$. Theorem follows after simplifying.

\section{B. Broadcast Efficiency}

Thus far, we have established the results relating to a specific packet transmitted by a single source with background interference. For broadcast efficiency, we are interested in the rate at which a node receives any packet successfully, instead of just the packet generated by a specific source. In this subsection, we will first derive the probability that a node receives any packet successfully when every node in the network broadcast a packet with probability $c$. We then show the rate at which nodes in the network receive packets successfully as a function of transmission probability $c$, node density $\lambda$, transmission power $p_{0}$, and packet size $L$.

In a large network, the carrier sensing mechanism of each node only reports whether any node in its vicinity is actively transmitting. Overall, the network remains asynchronous. To obtain the following result, however, we consider a specific instance when all the nodes perform carrier sensing at the same time, and concludes that the channel is free. Hence, for every node in the system, it transmits a packet with probability $c$, and it listens to a transmission with probability $1-c$.

Lemma 3: At a transmission opportunity, the probability that a node receives and decodes any packet successfully is

$$
P_{\text {s-any }}=c E[N] .
$$

Proof: Consider the ratio of expected number of received messages and expected number of vehicles.

To evaluate broadcast efficiency, or the amount of packets a node receive successfully in a unit duration regardless of the actual senders, we need to consider the time duration that a node transmits a packet $T_{\text {tx }}$, receives a packet $T_{\text {listen }}$, and is idle $T_{\text {idle. }}$. From IEEE 802.11p standards [17], we have

$$
\begin{aligned}
T_{\mathrm{tx}} & =T_{H}+L / R+T_{\mathrm{DIFS}}, \\
T_{\text {listen }} & \approx T_{H}+L / R+T_{\mathrm{DIFS}}, \\
T_{\text {idle }} & =T_{\text {slot }},
\end{aligned}
$$

where $T_{H}$ is the header transmission time, $T_{\mathrm{DIFS}}$ and $T_{\text {slot }}$ are a distributed inter-frame space and a slot duration, respectively.

Let $p_{\mathrm{cs}}$ be the carrier sensing threshold, the expected carrier sensing range ${ }^{2}$ is calculated by $d_{\mathrm{cs}}=\left(p_{0} / p_{\mathrm{cs}}\right)^{1 / \alpha}$. Then the probability that a node transmits, receives, and is idle can, respectively, be characterized as follows:

$$
\begin{aligned}
P_{\mathrm{tx}} & =c \\
P_{\text {idle }} & \approx(1-c)^{2 \lambda d_{\mathrm{cs}}}, \\
P_{\text {listen }} & =1-P_{\mathrm{tx}}-P_{\text {idle }} .
\end{aligned}
$$

The following theorem characterizes broadcast efficiency.

Theorem 2: The broadcast efficiency $U\left(c, \lambda, p_{0}, L\right)$ of a 1$\mathrm{D}$ broadcast wireless network is (in packets per second)

$$
U=\frac{1-c}{z^{1 / \alpha}} \cdot \frac{1-\exp \left(-2 c \lambda\left(p_{0} / n_{0}\right)^{1 / \alpha} \Gamma\left(1+\frac{1}{\alpha}\right)\right)}{T_{\mathrm{tx}}-\left(T_{\mathrm{tx}}-T_{\text {slot }}\right)(1-c)^{2 \lambda\left(p_{0} / p_{\mathrm{cs}}\right)^{1 / \alpha}}},
$$

and the average data rate that a node receives any packets successfully is

$$
R_{\mathrm{recv}}=U\left(c, \lambda, p_{0}, L\right) L \quad(\mathrm{bits} / \mathrm{sec}) .
$$

Proof: Simplify $U=\frac{P_{\text {s-any }}}{P_{\text {ix }} T_{\text {tx }}+P_{\text {idle }} T_{\text {idle }}+P_{\text {listen }} T_{\text {listen }}}$.

We now investigate the received data rate as $\lambda, p_{0}$ and $L$ vary in the following corollaries.

Corollary 1: As a function of payload size $L$, the average rate at which a node receives any packets successfully in a 1-D broadcast wireless network has the following asymptotic bound

$\lim _{L \rightarrow \infty} R_{\mathrm{recv}}=\frac{R(1-c)}{z^{1 / \alpha}} \cdot \frac{1-\exp \left(-2 c \lambda\left(p_{0} / n_{0}\right)^{1 / \alpha} \Gamma\left(1+\frac{1}{\alpha}\right)\right)}{1-(1-c)^{2 \lambda d_{\mathrm{cs}}}}$,

where $R$ is the transmission data rate.

Eq. (16) and the asymptotic bound in (17) for various payload sizes $L$ are compared in Fig. 1. Larger payload size improves the received data rate; however, the average number of packets received per unit time decreases as payload size increases.

Corollary 2: As a function of $\lambda p_{0}^{1 / \alpha}$, the average rate at which a node receives any packets successfully in a 1-D broadcast network has the following asymptotic bound

$$
\lim _{\lambda p_{0}^{1 / \alpha} \rightarrow \infty} R_{\mathrm{recv}}=\frac{(1-c) z^{-1 / \alpha}}{T_{\mathrm{tx}}} L .
$$

Eq. (16) and the asymptotic bound in (18) for various $\lambda p_{0}^{1 / \alpha}$ values are compared in Fig. 2. There might exist an optimal value for $\lambda p_{0}^{1 / \alpha}$ for known transmission probability $c$ and

\footnotetext{
${ }^{2}$ Due to fading, the carrier sensing range cannot be quantified by a constant value in general; however, we simplify the model and consider only its expected value, which is obtained by considering the macroscopic path loss.
} 
payload size $L$. Theorem 2 and Corollary 2 together show an important result for power control in VANET: with all other system parameters fixed, the same broadcast efficiency can be achieved by scaling $p_{0}^{1 / \alpha}$ inversely proportionally to $\lambda$.

\section{System Optimization}

From the last section, we investigated the interaction between the various parameters and the received data rate. In this section, we study the optimal probability of transmission $c$ for a system with known node density, transmission power and payload size. We also consider the case for dynamic networks, where the node density may vary rapidly within some bounds. The understanding of dynamic network is important VANET systems. For such networks, we provide a worst-case guaranteed strategy for finding the transmission probability that works extremely well for a wide range of density values.

\section{A. Optimal Transmission Probability}

Here, we assume that the node density $\lambda$ is known by all nodes. For instance, in a vehicular network, such density may be obtained from traffic surveillance cameras, and the result is broadcasted to all vehicles. In this case, the optimal transmission probability $\hat{c}$ can be readily obtained by considering the first order condition of (15), which leads to the following implicit solution:

$$
\begin{array}{r}
\frac{(1-\hat{c}) 2 \lambda \xi e^{-2 \lambda \hat{c} \xi}-\left(1-e^{-2 \lambda \hat{c} \xi}\right)}{(1-\hat{c}) 2 \lambda \xi e^{-2 \lambda \hat{c} \xi}+\left(2 \lambda d_{\mathrm{cs}}-1\right)\left(1-e^{-2 \lambda \hat{c} \xi}\right)} \\
=\left(1-\frac{T_{\text {slot }}}{T_{\mathrm{tx}}}\right)(1-\hat{c})^{2 \lambda d_{\mathrm{cs}}},
\end{array}
$$

where $\xi=\Gamma\left(1+\frac{1}{\alpha}\right) p_{0}^{1 / \alpha} n_{0}^{-1 / \alpha}$. Contrary to common assumptions [5], [13], [14], optimal broadcast efficiency cannot be achieved by setting transmission rate inversely proportional to the node density. The broadcast efficiency in (16) is plotted in Fig. 4 versus $c$ and we also show $\hat{c}(\lambda)$ as a monotonic decreasing function of $\lambda$ in Fig. 3.

\section{B. Worst-Case Guaranteed Transmission Probability}

In this subsection, we assume that it is impossible to estimate and distribute the node density accurately to the nodes in the network. Rather, the nodes know that the node density $\lambda \in\left[\lambda_{1}, \lambda_{2}\right]$, for $0<\lambda_{1}<\lambda_{2}$. In general, from Fig. 3, we see that the optimal transmission probability is density dependent.

We want to find a transmission probability that performs as close to the optimal broadcast efficiency as possible for any actual node density within the bounds. Hence, we find

$$
\hat{c}\left(\lambda_{1}, \lambda_{2}\right)=\arg \max _{c} \min \left\{\frac{U(c, \lambda)}{U(\hat{c}(\lambda), \lambda)}: \forall \lambda \in\left[\lambda_{1}, \lambda_{2}\right]\right\} .
$$

We call $0 \leq \frac{U(c, \lambda)}{U(\hat{c}(\lambda), \lambda)} \leq 1$ the normalized broadcast efficiency, which measures the efficiency with respect to what is optimally achievable. Note that the optimal transmission probability at exact node density $\lambda$ is $\hat{c}=\hat{c}(\lambda, \lambda)$.

From the optimal result, we know that the optimal transmission probability decreases as a function of $\lambda$, and it is easy to reason that the broadcast efficiency decreases as the transmission probability deviates from the optimal value for a given node density. Hence, the worst-case guaranteed transmission probability can be obtained by simply considering the interception of $\frac{U\left(c, \lambda_{1}\right)}{U\left(\hat{c}\left(\lambda_{1}\right), \lambda_{1}\right)}$ and $\frac{U\left(c, \lambda_{2}\right)}{U\left(\hat{c}\left(\lambda_{2}\right), \lambda_{2}\right)}$. This leads to the following implicitly solution:

$$
\begin{aligned}
& \frac{\left(1-e^{-2 \lambda_{2} \xi \hat{c}\left(\lambda_{1}, \lambda_{2}\right)}\right)\left(1-\left(1-\frac{T_{\text {slot }}}{T_{\text {tx }}}\right)\left(1-\hat{c}\left(\lambda_{1}, \lambda_{2}\right)\right)^{2 \lambda_{1} d_{c s}}\right)}{\left(1-e^{-2 \lambda_{1} \xi \hat{c}\left(\lambda_{1}, \lambda_{2}\right)}\right)\left(1-\left(1-\frac{T_{\text {slot }}}{T_{\text {tx }}}\right)\left(1-\hat{c}\left(\lambda_{1}, \lambda_{2}\right)\right)^{2 \lambda_{2} d_{c s}}\right)} \\
& =\frac{U\left(\hat{c}\left(\lambda_{2}\right), \lambda_{2}\right)}{U\left(\hat{c}\left(\lambda_{1}\right), \lambda_{1}\right)} .
\end{aligned}
$$

\section{Congestion Control}

Thus far, we have established the worst-case guaranteed transmission probability, and in the limit case the optimal transmission probability, given a range of possible node density values. The transmission probability is derived assuming the use of $p$-persistent CSMA protocol. However, many medium access control protocols today regulate the outgoing packets of a node through the use of contention window. For example, in IEEE 802.11p [17], the first packet in the queue uniformly chooses a backoff value between $[0, W-1]$, and the packet is only transmitted after the node senses a sufficient amount of channel inactivity. Nonetheless, the relationship between outgoing packet probability and contention window size is well known [13]. Let $\hat{c}\left(\lambda_{1}, \lambda_{2}\right)$ be the worst-case guaranteed transmission probability, then the corresponding worst-case guaranteed contention window size is $\hat{W}\left(\lambda_{1}, \lambda_{2}\right)=\left\lceil\frac{2}{\hat{c}\left(\lambda_{1}, \lambda_{2}\right)}-1\right\rceil$.

\section{Simulations}

We simulate highway scenarios in ns-2.33 [18], which has realistic wireless channel model, PHY and MAC layer abstractions, and is widely used in other simulation work [2], [5]. We adopt parameters (Table I) from WAVE/802.11p standards. The PHY layer monitors the accumulated interference level and adopts a SINR based capture model. Various vehicle densities and contention window sizes are evaluated.

Table I

SIMULATION PARAMETERS

\begin{tabular}{l|l||l|l}
\hline Central frequency & $5.890 \mathrm{Ghz}$ & Slot time & $13 \mathrm{us}$ \\
Channel bandwidth & $10 \mathrm{Mhz}$ & Header duration & $40 \mathrm{us}$ \\
Channel data rate & $3 \mathrm{Mbps}$ & Symbol duration & $8 \mathrm{us}$ \\
Modulation scheme & BPSK & Data packet size & 51 Bytes \\
Capture threshold for BPSK & $5 \mathrm{~dB}$ & Transmission power & $1 \mathrm{e}-5 \mathrm{Watt}$ \\
\hline
\end{tabular}

Fig. 5 plots the simulated normalized broadcast efficiency $(U / U(\hat{c}))$ and (15), when the accurate vehicle density is known. As we can see, the optimal contention window size matches well with the simulation result. More importantly, system designers may simply use the optimal contention window size derived in this paper.

In Fig. 6, we consider a wide range of vehicle densities $\lambda \epsilon$ $\{0.05,0.25,0.5\}(\mathrm{veh} / \mathrm{m})$, which corresponds to inter-vehicle spacings of 8,40 and $80(\mathrm{~m})$ on a 4-lane highway. The worstcase guaranteed contention window solution corresponds to the 


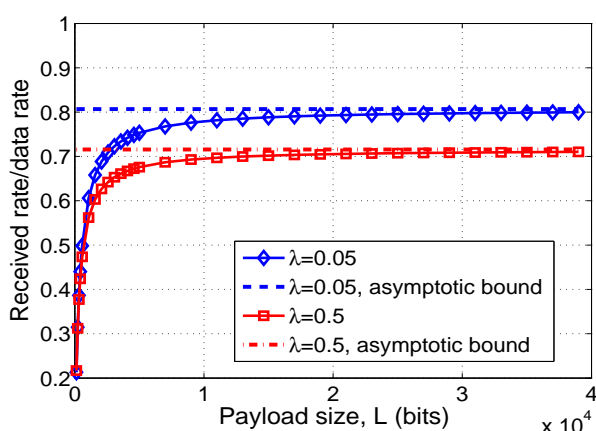

Figure 1 - Normalized received data rate as a function of $L$, with $p_{0}=10^{-5} \mathrm{~W}, c=0.05$.

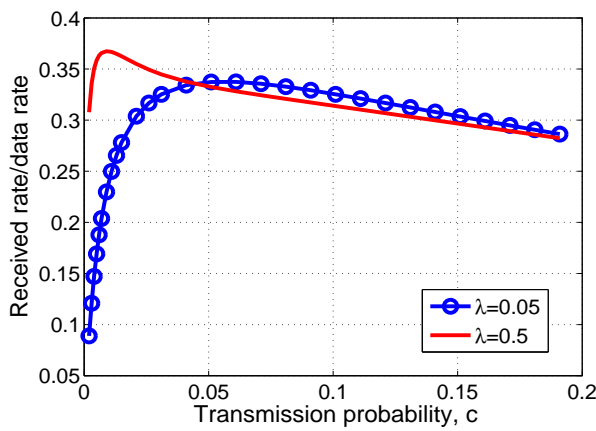

Figure 4. Received data rate versus transmission probability $c$, with $p_{0}=10^{-5} \mathrm{~W}, L=256$ bits.
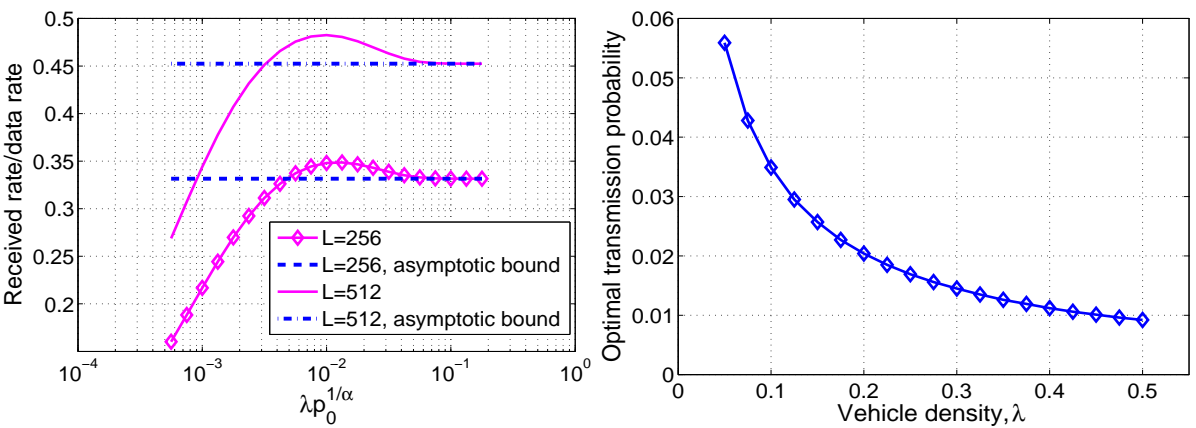

Figure 2. Normalized received data rate as a function of $\lambda p_{0}^{1 / \alpha}$, with $c=0.05$. function of $\lambda$, with $p_{0}=10^{-5} \mathrm{~W}, L=256$ bits.
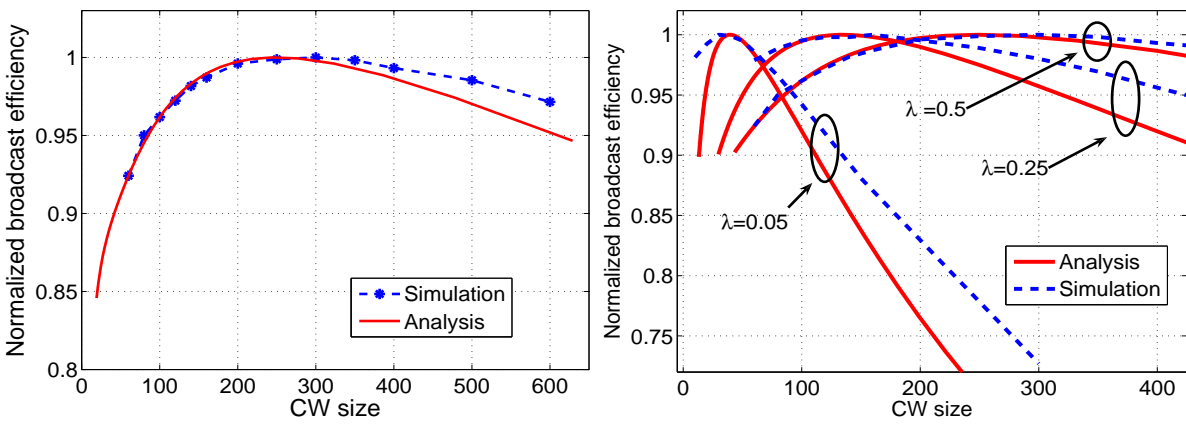

Figure 5. Normalized broadcast efficiency from analysis and simulation when density $\lambda=0.5$.
Figure 6. Normalized broadcast efficiency for a wide range of densities from analysis and simulation.
CW size at the interception of the curves for $\lambda=0.05$ and $\lambda=$ 0.5 . At $\mathrm{CW}=85$, the system guarantees that the broadcast efficiency is at least $95 \%$ of what is optimally achievable. If the system can further decides between $\lambda \in[0.25,0.5]$ (urban) and $\lambda \in[0.05,0.25]$ (rural), then $97 \%$ and $99 \%$ of the optimal performance can be guaranteed, respectively.

\section{CONCLUSION}

In this paper, we introduced broadcast efficiency to measure the average rate at which vehicles receive any broadcast packets successfully, and analyzed its behavior in Rayleigh fading channel with full probabilistic descriptions of signal and interference powers, and validated its accuracy using ns-2 simulations. The analysis provides the following fundamental results: (1) power control policy in VANET as node density varies; (2) the complete characterization of the optimal transmission probability as a function of node density, transmission power, packet length and other VANET system parameters; (3) a worst-case guaranteed congestion control strategy that achieves over $95 \%$ of the optimal performance even when the bounds on node density differs by an order of magnitude. This implies that a VANET system at most needs to spend only very little resource to conduct rough estimation of node density.

\section{REFERENCES}

[1] F. Li and Y. Wang, "Routing in Vehicular Ad Hoc Networks: A survey," IEEE Veh. Technol. Mag., vol. 2, no. 2, pp. 12-22, June 2007.

[2] M. Torrent-Moreno, S. Corroy, F. Schmidt-Eisenlohr, and H. Hartenstein, "IEEE 802.11-based one-hop broadcast communications: understanding transmission success and failure under different radio propagation environments," in Proc. of the 9th ACM MSWiM, 2006, pp. 68-77.

[3] R. Yim, J. Guo, P. Orlik, and J. Zhang, "Received Power-Based Prioritized Rebroadcasting for V2V Safety Message Dissemination," in Proc. of Int. Transport. Sys. World Congr., Sept. 2009.
[4] J. Yin, T. ElBatt, G. Yeung, B. Ryu, S. Habermas, H. Krishnan, and T. Talty, "Performance evaluation of safety applications over DSRC vehicular ad hoc networks," in Proc. of 1st VANET workshop, 2004.

[5] D. Jiang, Q. Chen, and L. Delgrossi, "Communication density: a channel load metric for vehicular communications research," in Proc. of MASS'07.

[6] J. Mittag, F. Schmidt-Eisenlohr, M. Killat, J. Härri, and H. Hartenstein, "Analysis and design of effective and low-overhead transmission power control for VANETs," in Proc. of the 5th VANET workshop, 2008.

[7] "ITS Std SAE J2735 - Dedicated Short Range Communications (DSRC) Message Set Dictionary," http://www.standards.its.dot.gov/.

[8] "IEEE Std 1609 family, IEEE Trial-Use Standard for Wireless Access in Vehicular Environments(WAVE)," Nov. 2006.

[9] M. Torrent-Moreno, D. Jiang, and H. Hartenstein, "Broadcast reception rates and effects of priority access in 802.11-based vehicular ad-hoc networks," in Proc. of the 1st VANET workshop, 2004, pp. 10-18.

[10] X. Ma, X. Chen, and H. Refai, "Performance and reliability of DSRC vehicular safety communication: a formal analysis," EURASIP Journal on Wireless Communications and Networking.

[11] M. Killat and H. Hartenstein, "An empirical model for probability of packet reception in vehicular ad hoc networks," EURASIP Journal on Wireless Communications and Networking.

[12] M. Zorzi and R. Rao, "Capture and retransmission control in mobile radio,” IEEE J. Select. Areas Commun, vol. 12, pp. 1289-1298, 1994.

[13] G. Bianchi, "Performance analysis of the IEEE 802.11 distributed coordination function," IEEE Journal on selected areas in communications, vol. 18 , no. 3 , pp. $535-547,2000$.

[14] F. Calì, M. Conti, and E. Gregori, "Dynamic tuning of the IEEE 802.11 protocol to achieve a theoretical throughput limit," IEEE/ACM Trans. on Networking (TON), vol. 8, no. 6, pp. 785-799, 2000.

[15] J. Zhu, B. Metzler, X. Guo, and Y. Liu, "Adaptive CSMA for scalable network capacity in high-density WLAN: a hardware prototyping approach," in Proc. of INFOCOM, 2006, pp. 1-10.

[16] L. Kleinrock and F. Tobagi, "Packet switching in radio channels: Part I-carrier sense multiple-access modes and their throughput-delay characteristics," IEEE Trans. on Communications, vol. 23, no. 12, pp. $1400-1416,1975$.

[17] "IEEE P802.11p/D5.0, IEEE 802.11 Amendment 7: Wireless Access in Vehicular Environments," Nov. 2008.

[18] "ns-2.33: the network simulator version 2.33," http://www.isi.edu/nsnam/ns. 